\title{
Shared-PPRM: A Memory-Efficient Representation for Boolean Reversible Functions
}

\author{
Yasaman Sanaee $^{\mathrm{a}}$, Mehdi Saeedi ${ }^{\mathrm{b}}$, Morteza Saheb Zamani ${ }^{\mathrm{b}}$ \\ Quantum Design Automation Lab \\ Amirkabir University of Technology, Computer Engineering Department, Tehran, Iran \\ Email: ${ }^{a} y$ sanaee@dena.aut.ac.ir, ${ }^{b}\{$ msaeedi,szamani\}@aut.ac.ir
}

\begin{abstract}
A memory-efficient representation scheme, sharedPPRM (SPPRM), for Boolean reversible functions is introduced and analyzed. Compared with conventional PPRM expansion, SPPRM reduces memory usages by using one memory location for many repetitive PPRM sub-expressions. To evaluate the effects of data structure on SPPRM representation, two linked listbased data structures are also examined. The experimental results show the efficiency of the proposed SPPRM representation for both memory usage and CPU time.
\end{abstract}

\section{Introduction}

Positive polarity Reed-Muller expansion (PPRM) has been used to represent reversible functions extensively. However, its memory usage could be too high which limits the applicability of algorithms. On the other hand, as the size of a reversible function increases, its truth table size grows exponentially. Therefore, an efficient data structure to reduce memory usages is vital.

\section{SPPRM Representation}

Since each $n$-variable reversible function $F$ has $n$ output variables, i.e. $f_{1}, f_{2} \ldots f_{n}$, the PPRM expansion of $F$ contains $n$ PPRM expressions each of which is the result of EXORing several, from 1 to $2^{n}$, subexpressions.

Definition 1: A given $n$-input $n$-output Boolean reversible function can be represented by a shared PPRM (SPPRM) representation if its PPRM expressions have common sub-expressions. In such a situation, the common sub-expressions are represented for all $n$ expressions only once.

Definition 2: Given an $n$-input, $n$-output Boolean reversible specification $F$ represented as Equation (1) with input variables $x_{1}, x_{2}, \ldots, x_{n}$, the memory cost for the (S)PPRM representation of $F$ is defined as Equation (2) where $a_{i, j} \in\{0,1\}$.

$$
\begin{aligned}
& f_{1}=a_{1,1} \oplus \ldots \oplus a_{1,2^{n}} x_{1} x_{2} \cdots x_{n} \\
& f_{2}=a_{2,1} \oplus \ldots \oplus a_{2,2^{n}} x_{1} x_{2} \cdots x_{n} \\
& \dddot{f_{n}}=a_{n, 1} \oplus \ldots \oplus a_{n, 2^{n}} x_{1} x_{2} \cdots x_{n} \\
& M C=\sum_{i=1}^{n} \sum_{j=1}^{2^{n}} a_{i, j}
\end{aligned}
$$

Theorem 1: Suppose an $n$-input $n$-output reversible specification $F$. Assume that an $m$-term $\left(m \Omega^{n}\right)$ subexpression $v$ is common among $k(k \leq n)$ output variables. Converting the PPRM expansion of $F$ to SPPRM expansion improves the memory cost if the following equation is held:

$$
m>\frac{k}{k-1}
$$

\section{The Effects of Data Structures}

To use SPPRM expansion, an efficient data structure is needed. In this section, two linked listbased data structures are introduced and analyzed. For each sub-expression of a PPRM expansion, a distinct linked list is considered as shown in the following example.

Example 1: Consider a given specification $\mathrm{F}=$ $(3,4,6,7,0,1,5,2)$. Fig. 1 illustrates the linked list representation of the PPRM expansion.

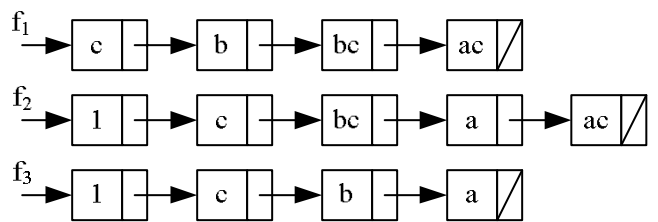

Fig. 1 Linked list-based data structure for PPRM expansion of Example (1) 
In order to use the linked list-based data structure for SPPRM representation, one may use a separate linked list for each common sub-expression and use its memory address for all related output variables as demonstrated in Fig. 2.

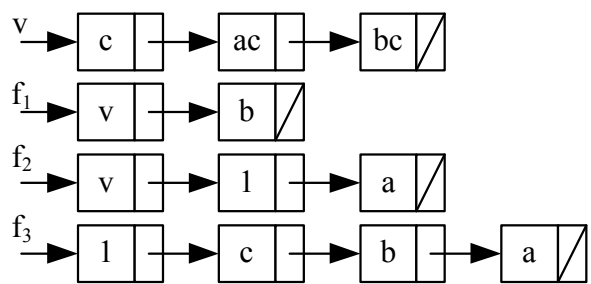

Fig. 2 Type-1 linked list-based data structure for SPPRM expansion of Example (1)

On the other hand, it may be possible to represent the common sub-expression for only one output variable with no internal node requirement. Other related output variables should refer it as shown in Fig. 3.

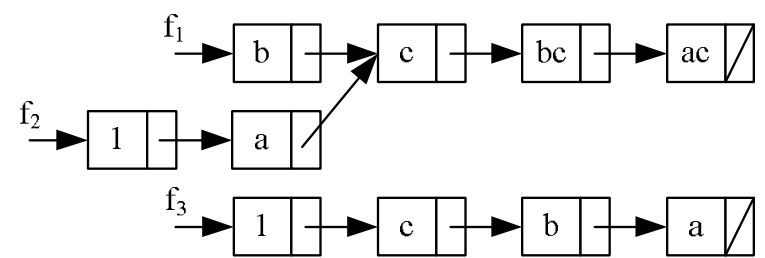

Fig. 3 Type-2 linked list-based data structure for SPPRM expansion of Example (1)

It can be verified that Type- 2 data structure is more efficient than Type-1 in terms of memory usage. Therefore, for the rest of this paper, Type-2 data structure is used to evaluate the efficiency of SPPRM representation.

While SPPRM could reduce memory usage inherently, SPPRM construction has a negligible timing overhead. In this section, a PPRM-to-SPPRM conversion algorithm is described. Using Type-2 data structure, the input of conversion algorithm is $n$ linked lists each of which contains some sub-expressions. The output of the algorithm is the resulted SPPRM expansion. Fig. 4 shows the SPPRM extraction algorithm. As illustrated in this figure, for each $m$ and $k$ values (see Theorem 1), all combinations of $k$ output variables are found and used to select the possible common sub-expression. In other words, for each combination, a common sub-expression is chosen and its memory usage improvement after SPPRM representation is found. After finding all possible common sub-expressions, the best one with the maximum memory usage improvement is selected and used to construct SPPRM expansion. The time complexity of the algorithm is $\mathrm{O}\left(2^{2 \mathrm{n}}\right)$.

\section{Experimental Results}

The proposed SPPRM expansion and the applied data structure were implemented in $\mathrm{C}++$ and all of the experiments were done on an Intel Pentium IV $3 \mathrm{GHz}$ computer with 1GB memory. To evaluate the efficiency of the SPPRM expansion, all 40320 3-input 3-output reversible Boolean functions were examined. In other words, the numbers of functions with common sub-expressions in their 2 or 3 output variables were calculated. In addition, the percentages of memory improvement after using SPPRM representation were extracted. The results of using SPPRM expansion for 3-input 3-output reversible functions are shown in Table 1. It can be verified that more than $16 \%$ improvements are obtained in memory usage with no considerable CPU overhead.

In addition, the results of using SPPRM expansion for 16 examples taken from the literature [4] were also shown in Table 2. As shown in this table, $18.5 \%$ improvement is achieved on average.

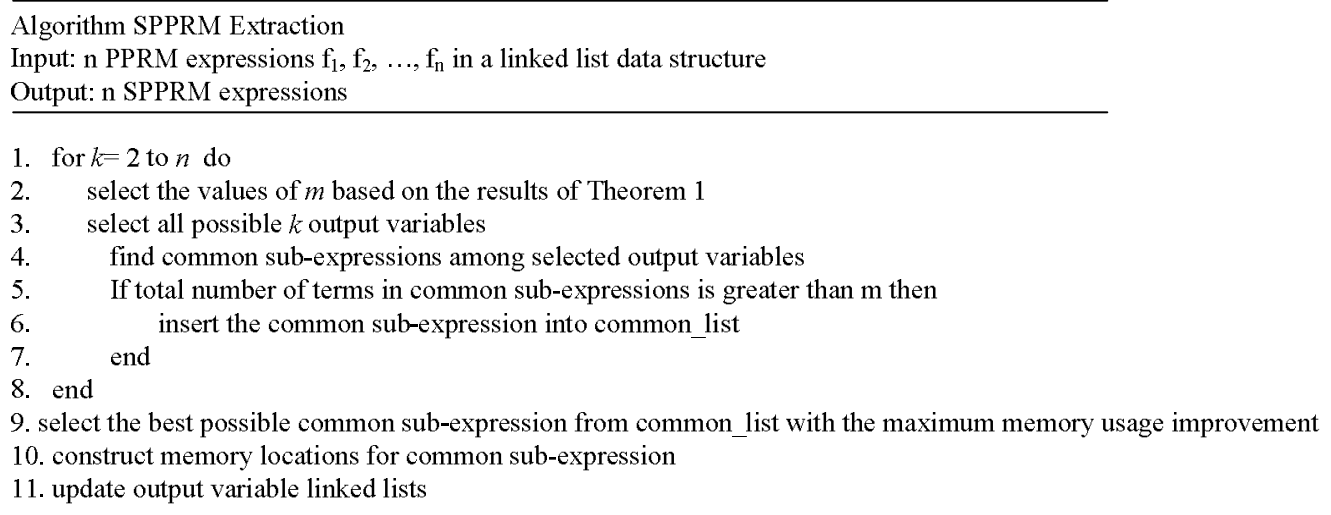

Fig. 4 SPPRM extraction algorithm 
Table 1- SPPRM Memory improvement for all 3input 3-output reversible functions

\begin{tabular}{|c||c|c|c|c|c|}
\hline \multirow{2}{*}{$\begin{array}{c}* \\
\text { \# of sub- } \\
\text { expressions }\end{array}$} & \multirow{2}{*}{ \# of functions } & \multirow{2}{*}{$\begin{array}{l}\text { \# of sub- } \\
\text { expressions }\end{array}$} & \multicolumn{2}{l|}{ \# of functions } \\
\cline { 2 - 5 } & PPRM & SPPRM & & PPRM & SPPRM \\
\hline 1 & 0 & 0 & 10 & 6426 & 9024 \\
\hline 2 & 0 & 0 & 11 & 7938 & 5220 \\
\hline 3 & 6 & 6 & 12 & 7440 & 2148 \\
\hline 4 & 72 & 108 & 13 & 5004 & 0 \\
\hline 5 & 324 & 612 & 14 & 2250 & 0 \\
\hline 6 & 870 & 1860 & 15 & 690 & 0 \\
\hline 7 & 1782 & 4446 & 16 & 180 & 0 \\
\hline 8 & 2916 & 7308 & 17 & 36 & 0 \\
\hline 9 & 4380 & 9588 & 18 & 6 & 0 \\
\hline
\end{tabular}

Table 2- SPPRM expansion for several examples [4]

\begin{tabular}{|c|c|c|c|c|}
\hline \multirow{2}{*}{ ckt \# } & \multirow{2}{*}{ Specification } & \multicolumn{3}{|c|}{ Memory usage } \\
\hline & & PPRM & SPPRM & Imp $\%$ \\
\hline 1 & $(1,0,3,2,5,7,4,6)$ & 8 & 8 & 0 \\
\hline 2 & $(7,0,1,2,3,4,5,6)$ & 10 & 6 & 40 \\
\hline 3 & $(0,1,2,3,4,6,5,7)$ & 7 & 7 & 0 \\
\hline 4 & $(0,1,2,4,3,5,6,7)$ & 13 & 7 & 46.15 \\
\hline 5 & $\begin{array}{c}(0,1,2,3,4,5,6,8,7,9,10 \\
11,12,13,14,15)\end{array}$ & 34 & 13 & 61.76 \\
\hline 6 & $(1,2,3,4,5,6,7,0)$ & 6 & 6 & 0 \\
\hline 7 & $\begin{array}{c}(1,2,3,4,5,6,7,8,9,10 \\
11,12,13,14,15,0)\end{array}$ & 8 & 8 & 0 \\
\hline 8 & $\begin{array}{c}(0,7,6,9,4,11,10,13,8 \\
15,14,1,12,3,2,5)\end{array}$ & 10 & 10 & 0 \\
\hline 9 & $(3,6,2,5,7,1,0,4)$ & 11 & 11 & 0 \\
\hline 10 & $(1,2,7,5,6,3,0,4)$ & 11 & 8 & 27.3 \\
\hline 11 & $(4,3,0,2,7,5,6,1)$ & 13 & 9 & 30.8 \\
\hline 12 & $(7,5,2,4,6,1,0,3)$ & 12 & 9 & 25 \\
\hline 13 & $\begin{array}{c}(6,2,14,13,3,11,10,7,0 \\
5,8,1,15,12,4,9)\end{array}$ & 24 & 21 & 12.5 \\
\hline 14 & $\begin{array}{c}(9,7,13,10,4,2,14,3,0 \\
12,6,8,15,11,1,5)\end{array}$ & 26 & 21 & 19.2 \\
\hline 15 & $\begin{array}{c}(6,4,11,0,9,8,12,2,15 \\
5,3,7,10,13,14,1)\end{array}$ & 29 & 24 & 17.2 \\
\hline 16 & $\begin{array}{c}(13,1,14,0,9,2,15,6,12 \\
8,11,3,4,5,7,10)\end{array}$ & 24 & 20 & 16.66 \\
\hline & averas & & & 18.53 \\
\hline
\end{tabular}

To further evaluate the cost of using SPPRM expansion, we examined 33 reversible benchmarks [6] the percentages of memory improvements of which are shown in Table 3. As shown in Table 3, 9.72\% improvement is achieved on average.

Table 3- The results of using SPPRM expansion for reversible benchmarks

\begin{tabular}{|c|c|c|c|c|}
\hline \multirow{2}{*}{$\begin{array}{c}\text { circuit } \\
\#\end{array}$} & \multirow{2}{*}{ Benchmark } & \multicolumn{3}{|c|}{ Memory usage } \\
\hline & & PPRM & SPPRM & Imp $\%$ \\
\hline 1 & 2 of5 & 45 & 32 & 22.2 \\
\hline 2 & 3 of17 & 14 & 10 & 28.8 \\
\hline 3 & $4 \bmod 5$ & 15 & 12 & 20 \\
\hline 4 & 4of49 & 39 & 30 & 23 \\
\hline 5 & $5 \bmod 5$ & 25 & 25 & 0 \\
\hline 6 & 5one 013 & 67 & 46 & 31.3 \\
\hline 7 & 5one245 & 111 & 86 & 22.5 \\
\hline 8 & 6one0246 & 12 & 12 & 0 \\
\hline 9 & 6one0135 & 11 & 11 & 0 \\
\hline 10 & alu & 55 & 49 & 10.9 \\
\hline 11 & $\operatorname{decod} 24$ & 30 & 24 & 20 \\
\hline 12 & graycode10 & 19 & 19 & 0 \\
\hline 13 & graycode20 & 39 & 39 & 0 \\
\hline 14 & graycode6 & 11 & 11 & 0 \\
\hline 15 & ham3 & 8 & 4 & 50 \\
\hline 16 & ham7 & 32 & 29 & 9.8 \\
\hline 17 & hwb4 & 28 & 22 & 21.4 \\
\hline 18 & hwb5 & 85 & 75 & 11.7 \\
\hline 19 & majority & 9 & 9 & 0 \\
\hline 20 & majority5 & 55 & 46 & 16.7 \\
\hline 21 & majority7 & 289 & 259 & 10.4 \\
\hline 22 & mod15adder & 23 & 23 & 0 \\
\hline 23 & mod32adder & 41 & 41 & 0 \\
\hline 24 & mod5adder & 82 & 82 & 0 \\
\hline 25 & mod64adder & 75 & 75 & 0 \\
\hline 26 & $\operatorname{rd} 32$ & 9 & 9 & 0 \\
\hline 27 & $\mathrm{rd53}$ & 30 & 30 & 0 \\
\hline 28 & $\operatorname{rd73}$ & 104 & 104 & 0 \\
\hline 29 & shift10 & 43 & 43 & 0 \\
\hline 30 & shift115 & 59 & 59 & 0 \\
\hline 31 & shift28 & 111 & 111 & 0 \\
\hline 32 & xor5 & 9 & 9 & 0 \\
\hline 33 & 2 of5 & 45 & 32 & 22.2 \\
\hline \multicolumn{4}{|c|}{ average } & 9.72 \\
\hline
\end{tabular}


To evaluate the scalability of the proposed SPPRM expansion, several other experiments were also performed. We generated 500 random circuits of different input sizes. Then, both PPRM and SPPRM expansions were constructed. The numbers of memory usages are reported in Table 4. As shown in Table 4, using SPPRM for reversible circuit representation leads to better memory usage with a negligible CPU overhead.

\section{Conclusion}

Today, reversible logic has received considerable attention in various research areas. In this paper, a memory-efficient representation scheme, SPPRM, for Boolean reversible functions was introduced and evaluated. In addition, the effects of various data structures on its efficiency were examined. Compared with the commonly used PPRM expansion, SPPRM could lead to a great improvement on memory usage.

\section{References}

Table 4- The results of using SPPRM expansion for random circuits of various sizes

\begin{tabular}{|c|c|c|c|c|c|c|c|c|c|c|c|c|}
\hline \multirow{3}{*}{ \# of sub-expressions } & \multicolumn{12}{|c|}{ \# of functions } \\
\hline & \multicolumn{2}{|c|}{ 4-input } & \multicolumn{2}{|c|}{ 5-input } & \multicolumn{2}{|c|}{ 6-input } & \multicolumn{2}{|c|}{ 7-input } & \multicolumn{2}{|c|}{ 8-input } & \multicolumn{2}{|c|}{ 9-input } \\
\hline & (P) & (SP) & (P) & (SP) & (P) & (SP) & $(\mathrm{P})$ & (SP) & (P) & (SP) & (P) & (SP) \\
\hline$[\mathrm{min}, \min +\mathrm{k})$ & 4 & 1 & 7 & 5 & 4 & 2 & 3 & 4 & 4 & 11 & 2 & 3 \\
\hline$[\min +\mathrm{k}, \min +2 \mathrm{k})$ & 4 & 7 & 12 & 8 & 23 & 13 & 3 & 5 & 22 & 25 & 8 & 16 \\
\hline$[\min +2 \mathrm{k}, \min +3 \mathrm{k})$ & 21 & 0 & 47 & 48 & 62 & 26 & 19 & 24 & 56 & 64 & 33 & 40 \\
\hline$[\min +3 \mathrm{k}, \min +4 \mathrm{k})$ & 37 & 38 & 71 & 55 & 119 & 87 & 44 & 71 & 106 & 107 & 64 & 74 \\
\hline$[\min +4 \mathrm{k}, \min +5 \mathrm{k})$ & 94 & 30 & 101 & 62 & 116 & 92 & 95 & 100 & 101 & 102 & 117 & 99 \\
\hline$[\mathrm{min}+5 \mathrm{k}, \min +6 \mathrm{k})$ & 103 & 130 & 93 & 133 & 93 & 133 & 97 & 114 & 123 & 101 & 100 & 117 \\
\hline$[\min +6 \mathrm{k}, \min +7 \mathrm{k})$ & 112 & 144 & 103 & 85 & 52 & 83 & 100 & 96 & 58 & 57 & 108 & 89 \\
\hline$[\min +7 \mathrm{k}, \min +8 \mathrm{k})$ & 79 & 65 & 42 & 68 & 26 & 38 & 89 & 57 & 20 & 18 & 47 & 44 \\
\hline$[\min +8 \mathrm{k}, \min +9 \mathrm{k})$ & 31 & 70 & 14 & 25 & 4 & 22 & 35 & 26 & 9 & 14 & 16 & 14 \\
\hline$[\min +9 \mathrm{k}, \max ]$ & 15 & 15 & 10 & 11 & 1 & 4 & 15 & 3 & 1 & 1 & 5 & 4 \\
\hline min & 18 & 14 & 61 & 53 & 163 & 143 & 394 & 362 & 955 & 1097 & 2191 & 2059 \\
\hline $\max$ & 39 & 30 & 94 & 77 & 222 & 189 & 479 & 435 & 890 & 1001 & 2396 & 2230 \\
\hline CPU overhead(sec) & & & & & & & & & & & & \\
\hline
\end{tabular}

- P and SP have been used as PPRM and SPPRM, respectively.

- $\mathrm{k}=(\max -\min ) / 10$

- 500 random circuits were generated for a given number of inputs
[1] R. Landauer, "Irreversibility and Heat Generation in the Computing Process,” IBM Journal, vol. 5, pp. 183-191, July 1961.

[2] C. Bennett, "Logical Reversibility of Computation," IBM Journal, vol. 17(6), pp. 525-532, November 1973.

[3] A. K. Prasad, V. V. Shende, I. L. Markov, J. P. Hayes, K. N. Patel, "Data Structures and Algorithms for Simplifying Reversible Circuits," ACM Journal on Emerging Technologies in Computing Systems, Vol. 2, Issue 4, 2006.

[4] M. Saeedi, M. Saheb Zamani, M. Sedighi, "On the Behavior of Substitution-Based Reversible Circuit Synthesis Algorithms: Investigation and Improvement," International Symposium on VLSI, 2007.

[5] P. Gupta, A. Agrawal, and N. K Jha, "An Algorithm for Synthesis of Reversible Logic Circuits," IEEE Transactions on Computer-Aided Design of Integrated Circuits and Systems, Vol. 25, Issue 11, pp. 2317-2330, 2006.

[6] D. Maslov, "Reversible Logic Synthesis," Ph.D. dissertation, The University of New Brunswick, 2003. 